\title{
Directed transport induced by $\alpha$-stable Lévy noises in weakly asymmetric periodic potentials
}

\author{
S. Risau-Gusman, S. Ibáñez, and S. Bouzat* \\ Consejo Nacional de Investigaciones Científicas y Técnicas, Centro Atómico Bariloche (CNEA), (8400) Bariloche, Río Negro, Argentina
}

(Received 3 October 2012; published 7 February 2013)

\begin{abstract}
We study the motion of a particle in spatially periodic potentials with broken mirror symmetry under the influence of white $\alpha$-stable Lévy noises. We consider both time-independent and fluctuating potentials. We focus on cases in which the spatial asymmetry of the potential is due not to a difference between the distances from an absolute minimum to the absolute maximum on its left and to the absolute maximum on its right but only to the curvatures of the potential profiles. The analysis is performed using the fractional Fokker-Planck formalism. Consistent results from Langevin simulations are also presented. We analyze the influence of the symmetry properties of the potentials in combination with the fluctuating characteristics of the system in the determination of the current. We find different situations in which both the absolute value and the direction of the current depend not only on the properties of the potential but also on the parameters characterizing the $\alpha$-stable Lévy noise. Among other features, we analyze the case of supersymmetric potentials. In particular, we show that a static supersymmetric potential produces no current, and we analyze the conditions for observing a nonvanishing current when the potential fluctuates between different supersymmetric profiles.
\end{abstract}

DOI: 10.1103/PhysRevE.87.022105

PACS number(s): 05.40.Fb, 05.10.Gg

\section{INTRODUCTION}

Random fluctuations as well as deterministic timedependent forces can induce directional transport in spatially periodic systems [1,2]. For this to happen, two main conditions are needed. First, the system has to be out of equilibrium. This indicates that thermal fluctuations alone are not enough. Second, an ingredient capable of inducing a preferred direction of motion is required. Such a source of asymmetry can come either from the spatial properties of the periodic system or from the temporal characteristics of the forces or fluctuations $[1,2]$.

The cases in which the asymmetry is given by the spatial properties of the system are usually modeled using anisotropic spatially periodic potentials, generically called ratchet potentials. Recently it has been shown [3,4] that an additive symmetric white $\alpha$-stable Lévy noise [3-6] is enough to induce directional transport of particles in a static ratchet potential. Thus, Lévy noises appear as a possible fluctuation source generating the needed nonequilibrium condition for transport. The case is interesting due to the ubiquitousness of Lévy noises in systems of very different origins [5] and particularly for possible applications to plasma physics [4]. This has motivated several studies providing further analysis and different generalizations of the Lévy ratchets, including the existence of inversions of current due to periodic modulation of the parameters [7], inertial effects [8], analysis of the weak noise regime [9], competition between Lévy forcing and a periodic ac driving [10], the effects of random flashing of the ratchet [11], and the space tempered Fokker-Planck equation [12].

White $\alpha$-stable Lévy noises have long tailed distributions that decay as power laws. At variance with what happens with Gaussian noise, particles moving in an environment with white Lévy noise have discontinuous trajectories [6]. The existence of instantaneous finite size jumps (which for symmetric Lévy noise are equiprobable in both directions) combined with the

*bouzat@cab.cnea.gov.ar asymmetry of the ratchet potential give rise to a nonvanishing current [9]. Importantly the lack of symmetry considered in previous studies [3,4,7-11] is identified with a difference between the distances from an minimum to the maximum on its left and to the maximum on its right. In this work we show that symmetry can be broken in more subtle ways and still produce a current. In particular, we explore potentials in which the minima are centered between two maxima (i.e., at the same distance from the two neighbor maxima) but the curvatures to each side differ. We show that a nonvanishing current can emerge in such kind of weakly asymmetric potentials under certain conditions. Conversely, we also show that not every nonsymmetrical potential is able to produce directed movement.

A different way to generate directed motion in the presence of noise is to introduce a temporal dependence in the potential. This dependence can be given by a continuous function of time or by a stochastic process. In this work we study the case in which the potential is randomly switched between different states with distinct properties of symmetry, focusing on the influence of weakly asymmetric profiles. Among other features, we analyze the case of supersymmetric potentials. We show that the only way to obtain a nonvanishing current by switching between supersymmetric potentials is to consider an irreversible switching process.

For the cases of both static and fluctuating potentials we observe that the direction of the current may depend on the parameters of the potentials and of the Lévy noise. Thus, several inversion effects are found in different situations. Most of our studies are performed using the fractional Fokker-Planck formalism that enables us to calculate the current of particles subject to periodic potentials in the presence of Lévy noise. We also provide consistent Langevin results for some selected examples.

The organization of the paper is as follows. In Sec. II we present the model and introduce the different types of potential profiles considered. In Sec. III we study transport in static potentials, while Sec. IV analyzes the case of fluctuating potentials. In Sec. V we present our conclusions. 

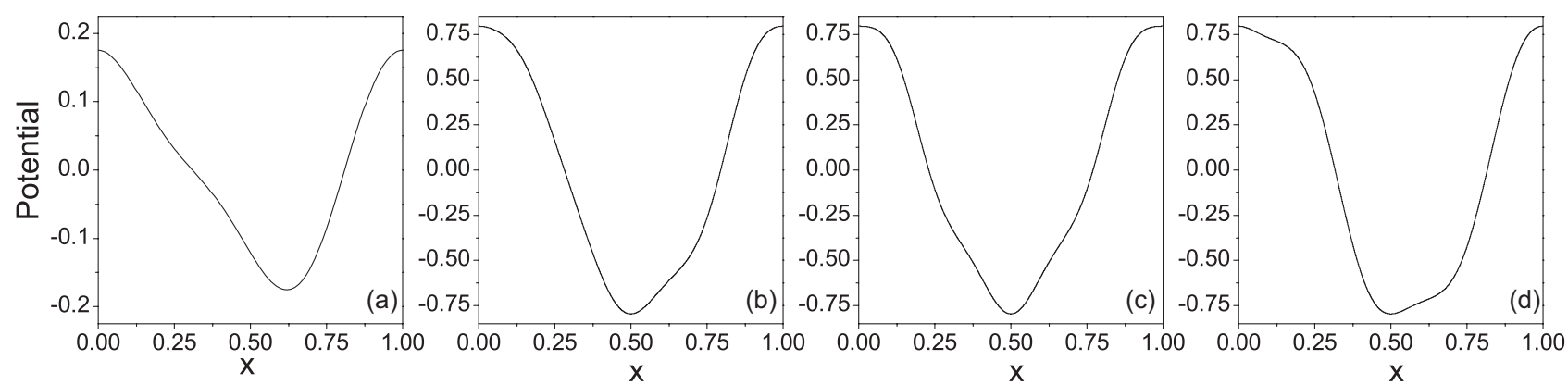

FIG. 1. Potential profiles. (a) Standard ratchet with asymmetric distances between maxima and minima. (b) Asymmetric nonsupersymmetric centered-minimum potential. (c) Symmetric centered-minimum potential. (d) Supersymmetric nonsymmetric potential [note the symmetry between the behavior in the region from $x=0$ to $x=0.5$ and that in the region $x=0.5$ to $x=1$, compare with panel (b)]. The potential in panel (a) is the one defined in Eq. (13) while the potentials in panels (b), (c), and (d) correspond to $V_{(A, B)}(x)$ defined in Eq. (7) with $(A, B)=(1,1 / 6),(0,1 / 3)$, and $(2,0)$, respectively.

\section{DYNAMICAL MODEL AND POTENTIALS}

We consider the overdamped movement of a particle under the influence of a time-dependent spatially periodic potential $[V(x, t)=V(x+L, t)]$ plus a white symmetric Lévy forcing, obeying the following Langevin equation:

$$
\frac{d x}{d t}=-V^{\prime}(x, t)+\xi_{\alpha, \chi}(t) .
$$

Here $-V^{\prime}(x, t)$ is the force derived from the potential $V(x, t)$, while $\xi_{\alpha, \chi}(t)$ is the white $\alpha$-stable symmetric Lévy noise $[4,13]$, defined by independent increments that follow a Lévy distribution whose characteristic function is $p_{\alpha}(k)=$ $\exp \left(-\chi|k|^{\alpha}\right)$. The parameters $0<\alpha \leqslant 2$ and $0<\chi$ are called respectively the stability index and the noise intensity of the white Lévy noise, and they determine the scale factor $\sigma=\chi^{1 / \alpha}$ of the noise $[4,5,8,13]$. Throughout the work we consider $\alpha>1$ so the first moment of the Lévy distribution is finite and the mean velocity of the particle results well defined [4]. In the limit $\alpha=2$ the statistics of the noise becomes Gaussian.

We focus on the analysis of the dynamics for different profiles and time dependencies of the potential $V(x, t)$ considering $L=1$. In the most general case, we consider $V(x, t)$ as given by the stochastic switching between a finite number of spatially periodic potentials $V^{j}(x)$ with $j=1, \ldots, M$. The transitions between the different potentials occur with constant rates. Most of the analysis will be done for the cases $M=1$ [timeindependent potentials $V(x, t)=V(x)], M=2$, and $M=3$. The profiles $V^{j}(x)$ are assumed to have vanishing mean value, so they can be classified according to their symmetry properties as follows. We will speak of a standard ratchet to refer to a potential for which, in a given spatial period between two absolute maxima, the absolute minimum is not equidistant from the two maxima. Conversely, a potential in which the absolute minimum is equidistant from the two absolute maxima will be referred to as a centered minimum potential. Within this group we can distinguish between symmetric potentials satisfying $V\left(x_{0}+x\right)=V\left(x_{0}-x\right)$ for some $x_{0}$ (i.e., potentials with reflection symmetry with respect to a certain position) and non symmetric centered minimum potentials. A special class of centered minimum potentials (which could be either symmetric or not) are the supersymmetric potentials [14], defined as those satisfying $V(x+L / 2)+V(x)=0$. In
Fig. 1 we show examples of the different potential types considered.

In order to calculate the current due to the dynamics of Eq. (1), in most of our work we consider the associated FokkerPlanck (FP) formalism, while Langevin simulations are only performed for some selected examples to check the results and show consistency. Assuming a stochastic switching between the potentials $V^{j}(x) j=1, \ldots, M$ with constant rates referred to as $\Gamma_{i k}$ for the transition from state $i$ to $k$, the system is represented by the following system of $M$ coupled FokkerPlanck equations [11] for the probability densities $P^{j}(x, t)$ of finding the system at state $j$ at time $t$, with particle at position $x$,

$$
\begin{aligned}
\partial_{t} P^{j}(x, t)= & \partial_{x}\left[P^{j}(x, t) \partial_{x} V^{j}(x)\right]+\chi \partial_{x}^{\alpha} P^{j}(x, t) \\
& +\sum_{l} \Gamma_{l j} P^{l}(x, t)-\Gamma_{j l} P^{j}(x, t),
\end{aligned}
$$

with $j=1, \ldots, M$. Here, $\partial_{x}^{\alpha}$ is the fractionary partial derivative defined as $\frac{\partial^{\alpha}}{\partial x}={ }_{-\infty} D_{x}^{\alpha}+{ }_{x} D_{-\infty}^{\alpha}$, with the operators $D^{\alpha}$ defined through their Fourier transforms,

$$
\begin{aligned}
\mathcal{F}\left({ }_{-\infty} D_{x}^{\alpha} f(x)\right) & =(-i k)^{\alpha} \tilde{f}_{k} \\
\mathcal{F}\left(_{x} D_{\infty}^{\alpha} f(x)\right) & =(i k)^{\alpha} \tilde{f}_{k}
\end{aligned}
$$

Here both $\mathcal{F}$ and the overline denote Fourier transformation with the prescription $g(x)=\sum_{j} \tilde{g}_{j}(t) e^{i k_{j} x}$, with $k_{j}=2 \pi j$ and integer $j$, for any arbitrary function $g(x)$ of spatial period $L=1$.

\section{TIME-INDEPENDENT POTENTIALS}

If the potential does not depend on time, there is a single state $V(x)$ and the Fokker-Planck equation (2) for the probability distribution $P(x, t)$ becomes

$$
\partial_{t} P(x, t)=\partial_{x}\left(P(x, t) \partial_{x} V(x)\right)+\chi \partial_{x}^{\alpha} P(x, t) .
$$

Developing $P(x, t)$ as a Fourier series, it is straightforward to obtain an evolution equation for the Fourier coefficients $\tilde{P}_{j}(t)[11]$. For the steady state, we define $\tilde{P}_{j} \equiv \lim _{t \rightarrow \infty} \tilde{P}_{j}(t)$ as the coefficients of the stationary distribution, which obey 

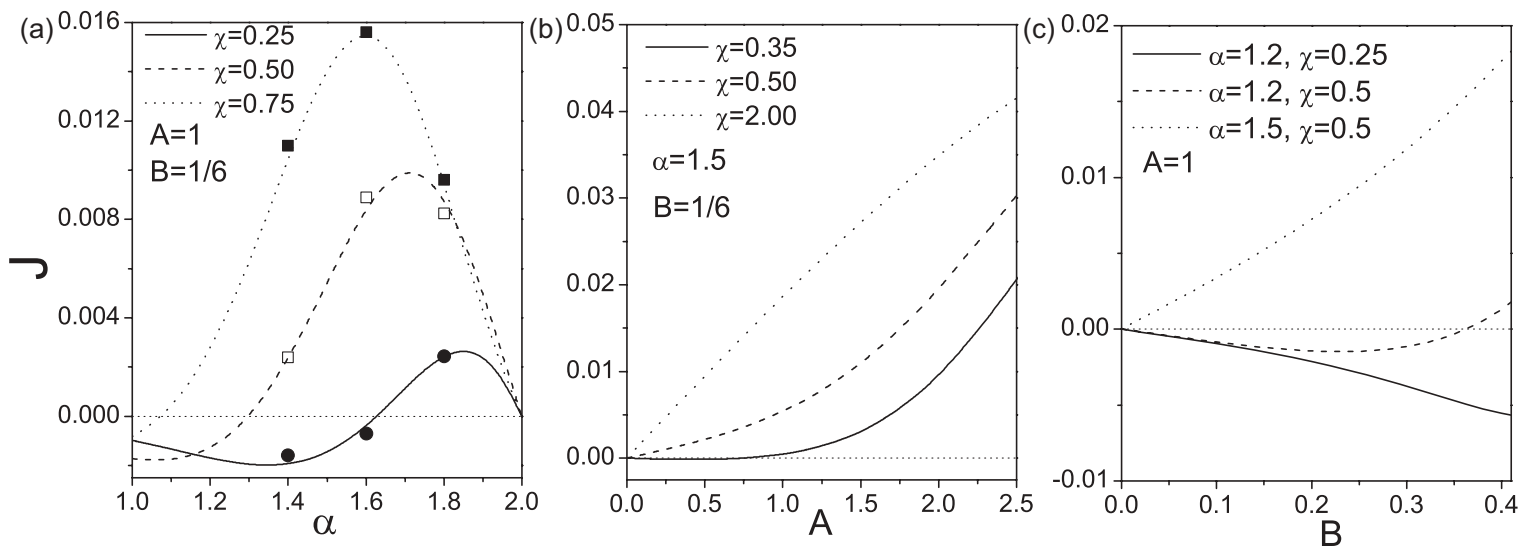

FIG. 2. (a) Current as a function of the exponent $\alpha$ of the Lévy noise, for particles placed in a potential $V_{(1,1 / 6)}(x)$ shown in Fig. 1(b). The lines correspond to the FP theory while symbols show results from Langevin simulations. (b) Current as function of the parameter $A$ of the potential for fixed $B$ and $\alpha$ and different values of noise intensity (results from the FP theory). (c) Current as function of the parameter $B$ for $A=1$ and different values of $\alpha$ and $\chi$ (results from the FP theory).

the following equations:

$$
-k_{j} \tilde{V}_{j}=\sum_{n \neq 0} k_{j-n} V_{j-n} \tilde{P}_{n}-\chi \operatorname{sgn}(j)\left|k_{j}\right|^{\alpha-1} \tilde{P}_{j}
$$

for $j \neq 0$, while the normalization of the probability demands that $\tilde{P}_{0}=1$. Equations (5) can in turn be written in matrix form as $\mathbf{M} \tilde{P}=v$, where the matrix $\mathbf{M}$ and the vector $v$ are defined, respectively, as $M_{i j}=k_{i-j} V_{i-j}-\chi \delta_{i j} \operatorname{sgn} j\left|k_{j}\right|^{\alpha-1}$ and $v=-k_{j} \tilde{V}_{j}$, with $i, j \in \mathbf{Z}$. $\delta_{i j}$ is a Kronecker $\delta$. Finally, the stationary current is defined as

$$
J=-i v^{\dagger} P,
$$

where the dagger denotes complex conjugation and transposition. In order to solve the matrix equation and compute the current, we consider a number of modes between 1000 and 5000 , depending on the values of $\alpha$ and $\chi$, and we check for convergence.

It is now well known [3,4] that for the case of a standard ratchet potential as that of figure 1(a) (i.e., not centered minimum), the preferred direction of motion is towards steepest mean slope of the potential. In other words, it points from the minimum of the potential towards the closest maximum. This is true independently of the values of $\alpha$ and $\chi$. Moreover, the current increase with the difference between the distances from the minimum to the left and right maxima [4]. Thus, the asymmetry of the potential, that manifests itself most clearly in the position of the minima with respect to the maxima, controls the direction of the current.

Here we analyze the case of centered minimum potentials for which, as we will show, the relation between asymmetry and current is far from straightforward. We consider the potential

$$
\begin{aligned}
V_{(A, B)}(x)= & \frac{1}{2 \pi}\left\{A\left[\sin (2 \pi x)-\frac{\sin (6 \pi x)}{3}\right]+5 \cos (2 \pi x)\right. \\
& +B[\cos (4 \pi x)-\cos (8 \pi x)]\} .
\end{aligned}
$$

We restrict the domain of $A$ and $B$ so the potential is centered minimum with absolute maxima at $x=0$ and $x=1$ and absolute minimum at $x=1 / 2$. For this, we need $|12 B|<$ 5 and $0 \leqslant A<A_{0}$, where $A_{0} \sim 2.5$ is a nonanalytic value slightly dependent on $B$. Importantly, $V_{(A, B)}(x)$ is symmetric (even) for $A=0$ and supersymetric for $B=0$. Some examples of the profiles of $V_{(A, B)}(x)$ are shown in Figs. 1(b)-1(d).

Figure 2 shows the current for this potential considering different values of $A, B, \alpha$, and $\chi$, computed from the FP formalism. In Fig. 2(a) we see that, for the nonsymmetric nonsupersymmetric centered-minimum potential shown in Fig. 1(b), both the absolute value and the direction of the current depend on $\alpha$ and $\chi$. Note that the shape of the potential favors the particle to locate preferably in the region $x>0.5$. This leads to a positive current in most situations, since escapes from the potential well to the right would result more probable than escapes to the left. However, small-enough values of $\alpha$ and $\chi$, which favor the existence of relatively infrequent but very large impulses, can invert the sign of the current. Figure 2(b) shows how the current vanishes for $A \rightarrow 0$, i.e., when the potential becomes symmetric. Meanwhile, in Fig. 2(c) we see that the current also vanishes when the potential becomes supersymmetric $(B=0)$. Using the the FP theory it is straightforward to check that for a symmetric potential the probability function is also symmetric, which gives a vanishing current. This fact is, however, well known and physically obvious. More interesting is the case of supersymmetric potentials. As we see below, the FP theory allows us to show that the current vanishes for all supersymmetric potentials. Thus, the example analyzed in Fig. 2(c) is a particular case of such a general result.

\section{A. Supersymmetric potentials}

To show that the particle current vanishes in a supersymmetric potential, we start by showing that, for any potential, when we change the sign of $\chi$ in Eq. (5), the current given by Eq. (6) does not change. Notice that the fact that negative values of $\chi$ have no clear physical meaning does not invalidate the mathematical argument that follows, concerning the matrix equations. Thus, we want to show that $J^{*}=J$, where the star symbol denotes the quantity obtained when the replacement 
$\chi \rightarrow-\chi$ is made. For this, note that the matrix $\mathbf{M}$ satisfies $\mathbf{M}^{*}=-\mathbf{M}^{\dagger}$. Then, using the definitions of $J, P$, and $\mathbf{M}$ we obtain

$$
\begin{aligned}
J^{*} & =-i v^{\dagger} P^{*}=-i v^{\dagger}\left(\mathbf{M}^{-1}\right)^{*} v=i v^{\dagger}\left(\mathbf{M}^{-1}\right)^{\dagger} v \\
& =\left(-i v^{\dagger} \mathbf{M}^{-1} v\right)^{\dagger}=J^{\dagger}=J .
\end{aligned}
$$

This result is completely independent of the potential. Now we show that, for the particular case of a supersymmetric potential, it is also true that $J^{*}=-J$, which in turn implies that the current vanishes. Note, first, that when the potential is supersymmetric it satisfies $\tilde{V}_{j}=0$ for all even values of $j$. Let us now define vectors $P_{e}$ and $P_{o}$ as the vectors that contain respectively the even and odd components of $P$ [i.e., $\left(P_{e}\right)_{j}=$ $P_{2 j}$ and $\left(P_{o}\right)_{j}=P_{2 j-\operatorname{sgn} j}$, with $j \neq 0$ ]. Doing the same for $v$ the supersymmetry of the potential implies $v_{o}=0$. Thus, in this case the matrix equation can be written as:

$$
\begin{aligned}
& M_{1} P_{e}-\chi M_{2} P_{o}=v_{o} \\
& M_{1} P_{o}-\chi M_{2} P_{e}=0
\end{aligned}
$$

and the current is $J=-i \bar{v} \tilde{P}_{o}$. If $\left(\tilde{P}_{o}, \tilde{P}_{e}\right)$ is a solution of Eq. (9) it is straightforward to see that if we perform the change $\chi \rightarrow$ $-\chi$, the solution of the new system satisfies $\left(\tilde{P}_{o}^{*}, \tilde{P}_{e}^{*}\right)=\left(\tilde{P}_{o},-\right.$ $\left.\tilde{P}_{e}\right)$. In turn, this implies that $J^{*}=-J$. Combining this with Eq. (8) we arrive at the conclusion that for supersymmetric potentials $J \equiv 0$.

\section{FLUCTUATING POTENTIALS}

\section{A. Switching between two potentials}

In this section we consider a potential that switches stochastically between two different states $V^{A}(x)$ and $V^{B}(x)$. The rate of the transition from state $A$ to state $B$ and back are $\Gamma_{A B}$ and $\Gamma_{B A}$, respectively. In this case, Eq. (2) becomes

$$
\begin{aligned}
\partial_{t} P^{A}(x, t)= & \partial_{x}\left[P^{A}(x, t) \partial_{x} V^{A}(x)\right]+\chi \partial_{x}^{\alpha} P^{A}(x, t) \\
& +\Gamma_{B A} P^{B}(x, t)-\Gamma_{A B} P^{A}(x, t), \\
\partial_{t} P^{B}(x, t)= & \partial_{x}\left[P^{B}(x, t) \partial_{x} V^{B}(x)\right]+\chi \partial_{x}^{\alpha} P^{B}(x, t) \\
& +\Gamma_{A B} P^{A}(x, t)-\Gamma_{B A} P^{B}(x, t) .
\end{aligned}
$$

Analogously to what was done for the constant potential case, we can found matrix equations for the Fourier components $\tilde{P}_{j}^{A}$ and $\tilde{P}_{j}^{B}$ of the stationary distributions. The method was presented in detail in Ref. [11]. The coefficients $\tilde{P}_{0}^{A}$ and $\tilde{P}_{0}^{B}$ represent the fraction of time that the potential spends in states A and B. Solving the corresponding steady-state equations, we obtain

$$
\tilde{P}_{0}^{A}=\frac{\Gamma_{B A}}{\Gamma_{A B}+\Gamma_{B A}}, \quad \tilde{P}_{0}^{B}=\frac{\Gamma_{A B}}{\Gamma_{A B}+\Gamma_{B A}} .
$$

Using this, the remaining steady-state equations can be written as single matrix equation,

$$
\left(\begin{array}{l}
v^{A} \\
v^{B}
\end{array}\right)=\left(\begin{array}{ll}
\mathbf{M}^{A A} & \mathbf{M}^{A B} \\
\mathbf{M}^{B A} & \mathbf{M}^{B B}
\end{array}\right) \cdot\left(\begin{array}{c}
\tilde{\tilde{P^{A}}} \\
\tilde{P^{B}}
\end{array}\right) .
$$

where $v_{j}^{A}=-k_{j} \tilde{V}_{j}^{A}$ and $v_{j}^{B}=-k_{j} \tilde{V}_{j}^{B}$, and the matrices are defined as $M_{i j}^{A B}=M_{i j}^{B A}=\Gamma_{A B}+\Gamma_{B A}, M_{i j}^{A A}=\left[k_{i-j} V_{i-j}^{A}-\right.$ $\left.\delta_{i j}\left(\chi \operatorname{sgn} j\left|k_{j}\right|^{\alpha-1}-\Gamma_{A B}\right)\right] / P_{0}^{A}$ and similarly for $M^{B B}$. This equation can be more compactly rewritten as $\mathbf{M} \tilde{P}=v$. Using this, the current can be written as $J=-i \bar{v} P$.

As mentioned in the previous section, it is well known $[3,4]$ that a white symmetric Lévy noise generates a positive current if a particle is placed on the standard ratchet potential,

$$
V(x)=\frac{1}{2 \pi} \sin (2 \pi x)+\frac{1}{8 \pi} \sin (4 \pi x),
$$

shown in Fig. 1(a). Now, using the theory just developed, it is interesting to see what happens when this potential is "decomposed" into the two symmetric components:

$$
\begin{aligned}
& V^{A}(x)=\frac{1}{2 \pi} \sin (2 \pi x) \\
& V^{B}(x)=\frac{1}{8 \pi} \sin (4 \pi x),
\end{aligned}
$$

which are switched at a rate $\Gamma_{A B}=\Gamma_{B A}=\Gamma$. Figure 3 shows the current for this case comparing it with what is obtained by switching, with the same rate, between the potential given by Eq. (13) and $V(x)=0$. This latter problem was analyzed in detail in Ref. [11].

As expected, in the slow switching limit, the system that switches between $V^{A}(x)$ and $V^{B}(x)$ produces zero current since the particle is able to adiabatically reach the steady state in each of the symmetric potentials [see Figs. 3(a)-3(c)]. In contrast, for the system in which the potential $V(x)$ is flashed (turned on an off), a nonvanishing current is observed at small $\Gamma$, whose value is half that obtained for the system with constant potential $V(x)$ [11]. This is because the particle is, half of the time, subject to a constant asymmetric potential. Meanwhile, in the fast switching limit, the current for both systems tends to the same value, which coincides with that obtained for a static potential equal to $V(x) / 2$ (i.e., with half amplitude), as was shown for the case of flashing in Ref. [11].

Interestingly, when switching between $V^{A}(x)$ and $V^{B}(x)$, a current inversion appears for $\alpha$ close to 2 [see Figs. 3(c) and 3(d)]. For the case of Gaussian noise (i.e., $\alpha=2$ ), the effect was previously reported in Ref. [15]. In that work, the emergence of a current for a system that switches between two symmetric potentials at equal rates has been linked to the fact that the maxima and minima of both potentials are shifted relatively to each other. This is certainly true and it is easy to see that switching between symmetric potentials whose maxima coincide cannot produce directed movement in the presence of Gaussian noise, if there is no more than one maximum per period. This is also true for any Lévy noise in general (i.e., $\alpha<2$ ). Notice that when a potential is symmetric there is a coordinate system in which it is an even function of $x$. In the case of $V^{A}(x)$ and $V^{B}(x)$, if the positions of the maxima coincide, it is possible to shift the common coordinates such that both potentials are even. Thus, the Fourier components of both functions have only real components which, in analogy to what was shown in the last section, implies that both particle distributions have that same property, which in turn implies that the current vanishes. If at least one potential has more than one maximum per period, the current will only vanish if the maxima or minima that coincide are those respect to which the functions are even.

In different contexts, the emergence of a current when two potentials are randomly switched, in the presence of Gaussian noise, has been associated to a shift in the position 

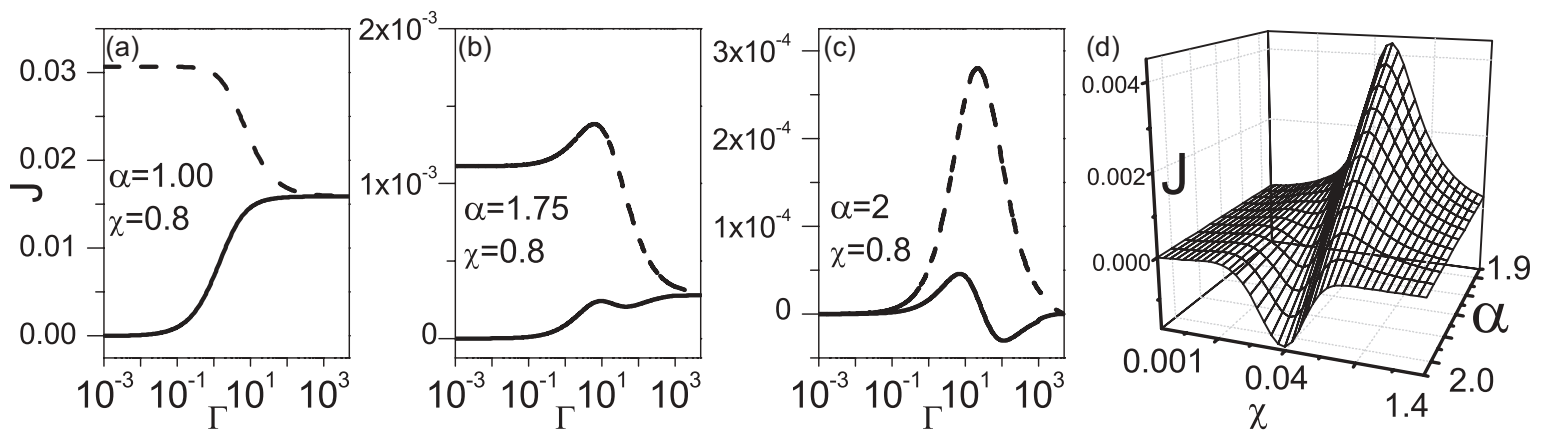

FIG. 3. Current as a function of the transition rate for a system that switches between the two sinusoidal functions in Eq. (14) [solid lines in panels (a), (b), and (c)] and for a system that switches between the standard ratchet in Eq. (13) and $V(x)=0$ [dashed lines in panels (a), (b), and (c)]. Panel (d) shows the current as a function of $\alpha$ and $\chi$ for a system that switches between the two sinusoidal functions in Eq. (14) with rate $\Gamma=10$.

of the minima [15] or to a difference in potential depth [16]. However, it is easy to see that none of these conditions is necessary to produce a current. Consider, for example, a particle moving in a potential that is being randomly switched between the potentials $V_{(0,1 / 3)}(x)$ [Fig. 1(c)] and $V_{(2,0)}(x)$ [Fig. 1(d)] defined in Eq. (7). Note that both potentials have centered minimum profiles with the same amplitude. As indicated, $V_{(0,1 / 3)}(x)$ is symmetric, whereas $V_{(2,0)}(x)$ is a supersymmetric (nonsymmetric) potential. This implies that no current is produced when particles are placed in any of these potentials under the influence of Lévy noise. However, the situation changes when these potentials are temporally switched, as shown in Fig. 4. When the switching is slow, no current is generated, as is to be expected. For larger values of switching the current depends on the type of Lévy noise affecting the particles. Interestingly, the noise can move the particles to the right or to the left, depending on the value of $\alpha$. When the switching is very fast, the particles can only "see" a temporal average of the potential, which coincides with the nonsymmetric nonsupersymmetric centered minimum potential $V_{(1 / 6,1)}(x)$ analyzed in the previous section (see Fig. 3).

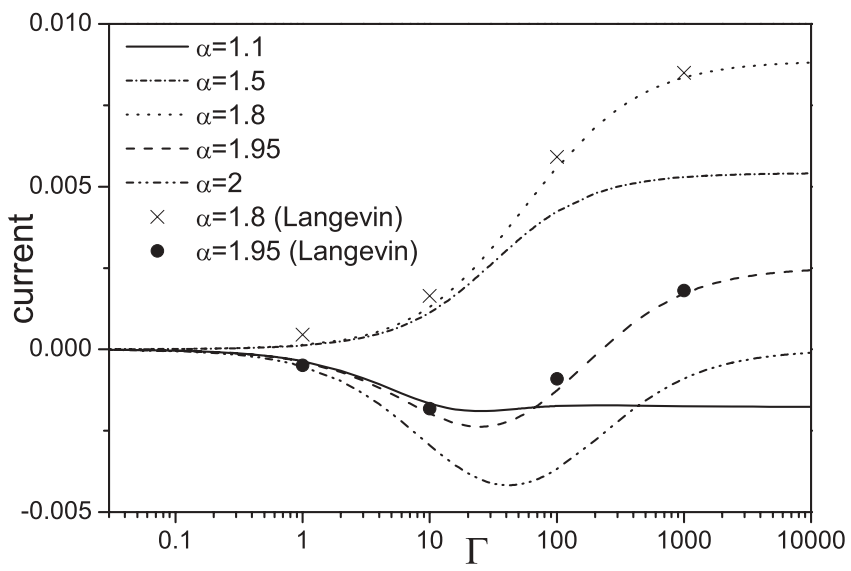

FIG. 4. Current as a function of $\Gamma$, the switching rate of the potentials, for different values of $\alpha$ and $\chi=0.5$. The switching takes place between the potentials $V_{(0,1 / 3)}(x)$ and $V_{(2,0)}(x)$. All curves correspond to FP results while the symbols indicate results from Langevin simulations for some selected examples.

\section{B. Switching between supersymmetric potentials}

Even though switching can produce directed movement even in the case of potentials that do not produce such movement when applied separately, this does not happen for some potentials. One interesting case is the family of supersymmetric potentials. Using the previous definitions, and using the fact that matrix $\mathbf{M}$ has the property that $\mathbf{M}^{*}=-\mathbf{M}^{\dagger}$, the proof that $J \equiv 0$ when $V^{A}$ and $V^{B}$ are supersymmetric is very similar to the one for a fixed, supersymmetric potential given in the previous section. This generalizes to all Lévy noises the result already known for Gaussian noise [14]. Notice that this result does not depend on the switching rates $\Gamma_{A B}$ and $\Gamma_{B A}$.

\section{Switching between more than two potentials}

The case of a potential switching between $M>2$ states $V^{j}(x)(j=1, \ldots, M)$ is very similar to what has been described in the previous sections but has a few important differences. The constants $\tilde{P}_{0}^{j}$, that represent the fraction of time spent by the potential in each state $j$ is given by the set of equations

$$
\sum_{l \neq j}\left(\Gamma_{l j} \tilde{P}_{0}^{l}-\Gamma_{j l} \tilde{P}_{0}^{j}\right)=0
$$

with $j=1, \ldots, M$, together with the normalization condition $\sum_{j} \tilde{P}_{0}^{j}=1$. Using the same proof as before, it can be shown that switching between supersymmetric potentials does not produce any current if the stochastic process is time reversible, i.e., if the rates satisfy the equality $\Gamma_{l j} \tilde{P}_{0}^{l}=\Gamma_{j l} \tilde{P}_{0}^{j}$ for every pair of states $V^{j}(x)$ and $V^{l}(x)$. This is equivalent to demanding that detailed balance is satisfied for all possible transitions. Notice that this restriction is trivial in the case of two states, because stochastic processes with only two states are always time reversible. Thus, in the general case, switching between more than two supersymmetric potentials can generate a current.

Interestingly, if the switching happens between symmetric potentials whose maxima coincide, not even a time asymmetry in the switching is able to produce directed movement.

In the previous section we have shown that a system with white Lévy noise, switching between two potentials, generates a current in the infinitely fast limit, because it is equivalent to having the system in a static potential that is the average of the ones being switched. In the infinitely slow limit it is 


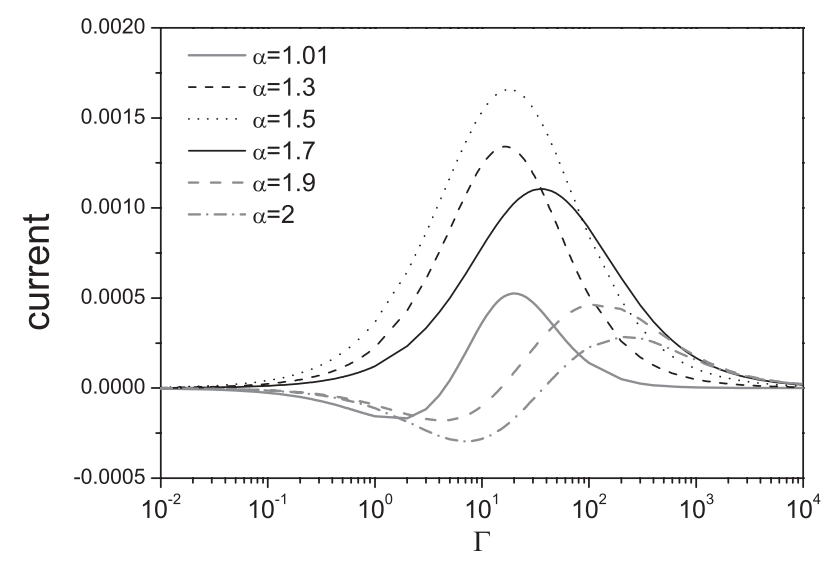

FIG. 5. Current as a function of $\Gamma$, for a system with three potentials stochastically switched for $\chi=1$ and several values of $\alpha$. The switching takes place between the potentials $V_{A}(x)=V_{(0,1 / 3)}(x)$, $V_{B}(x)=V_{(2,0)}(x)$, and $V_{C}(x)=V_{(0,-1 / 3)}(x)$ at rates $\Gamma_{A B}=\Gamma_{B A}=$ $2 \Gamma$ and $\Gamma_{A C}=\Gamma_{C A}=\Gamma_{B C}=\Gamma_{C B}=\Gamma$.

possible to have no current if the potentials being switched do not generate a current in the static case. In principle, it is not possible to have a vanishing current in both limits, as happens with Gaussian noise, when switching between only two potentials with Lévy noise. However, this effect can be achieved by combining three potentials, as seen in Fig. 5. Two of these potentials are symmetric, whereas the third is supersymmetric. The average of the three gives $V(2 / 3,5,0)$ which is supersymmetric. In this case, current inversions are observed for Lévy noises with $\alpha$ close to 1 and $\alpha$ close to 2 but not for intermediate values.

\section{SUMMARY AND CONCLUSIONS}

We have studied the transport of particles on weakly asymmetric spatially periodic potentials forced by white symmetric $\alpha$-stable Lévy noises. We have analyzed both the case of constant potentials and that of a fluctuating potential which stochastically switches between different profiles.

In the case of time-independent centered minimum potentials (i.e., potentials in which a given minimum is equidistant from the maximum on its right and that on its left), we have found that the direction of the current depends on the parameters $\alpha$ and $\chi$ characterizing the Lévy noise. This contrasts to what is found in standard ratchets, for which the current points always in the direction of the steepest slope of the potential, independently of the Lévy noise parameters.

Another important difference between the transport in static centered minimum potentials and standard ratchets concerns the weak noise limit $(\chi \rightarrow 0)$. In Ref. [9] it was shown that for $\chi \rightarrow 0$ the current is given by $J \simeq k_{\alpha} \chi[\zeta(\alpha, 1-q)-\zeta(\alpha, q)]$, where $\zeta(a, b)$ is the Hurwitz $\zeta$ function, $q$ is the distance from the minimum of the potential to the maximum that lies on its left, and $k_{\alpha}=\Gamma(\alpha) \sin (\pi \alpha / 2) / \pi$. Thus, note that while for standard ratchets the current grows linearly with $\chi$, in the case of centered minimum potentials analyzed in this paper the current should grow more slowly, since we have $q=1-q=$ $1 / 2$ and this makes the $\zeta$ functions cancel each other.

Concerning systems with fluctuating potentials, we have shown the existence of various situations in which it is possible to obtain a nonvanishing current by switching between weakly asymmetric potentials that do not produce current in the constant-profile case. We have also shown that, as happens for the constant potential case, when the potential switches between different weakly asymmetric profiles, both the magnitude and the direction of the current depend not only on the characteristics of the potentials but also on the parameters defining the Lévy noise. In particular, current inversions can be obtained by modifying only the value of $\alpha$ or $\chi$. In the fast switching limit, the current is equal to that obtained by considering a constant potential equal to average of the potentials considered, weighted accordingly to the transitions rates among them.

Our results on supersymmetric potentials with Lévy noises generalize those given in Ref. [14] for Gaussian noises. We have shown analytically that a constant supersymmetric potential does not produce current and that the only way to obtain a nonvanishing current by switching between supersymmetric potentials is to consider an irreversible switching process. This requires at least three different supersymmetric potentials.

\section{ACKNOWLEDGMENTS}

The authors acknowledge A. B. Kolton for interesting discussion and support from CNEA and CONICET under Grant No. PIP 11220080100076.
[1] P. Reimann, Phys. Rep. 361, 57 (2002).

[2] P. Hänggi and F. Marchesoni, Rev. Mod. Phys. 81, 387 (2009).

[3] B. Dybiec, E. Gudowska-Nowak, and I. M. Sokolov, Phys. Rev. E 78, 011117 (2008).

[4] D. del-Castillo-Negrete, V. Yu Gonchar, and A. V. Chechkin, Physica A 387, 6693 (2008).

[5] G. Samorodnitsky and M. S. Taqqu, Stable Non-Gaussian Random Processes (Chapman \& Hall, New York, 1994); D. Applebaum, Lévy Processes and Stochastic Calculus (Cambridge University Press, Cambridge, 2004).

[6] K. Jacobs, Stochastic Processes for Physicists (Cambridge University Press, Cambridge, 2010).

[7] B. Dybiec, Phys. Rev. E 78, 061120 (2008).

[8] S. Bouzat, Physica A 389, 3933 (2010).
[9] I. Pavlyukevich, B. Dybiec, A. V. Chechkin, and I. M. Sokolov, Eur. Phys. J. Spec. Top. 191, 223 (2010).

[10] Bao-quan Ai and Ya-feng He, J. Stat. Mech. (2010) P04010.

[11] S. A. Ibáñez, A. B. Kolton, S. Risau-Gusman, and S. Bouzat, J. Stat. Mech. (2011) P08025.

[12] A. Kullberg and D. del Castillo Negrete, J. Phys. A 45, 255101 (2012).

[13] B. Dybiec, E. Gudowska-Nowak, and P. Hänggi, Phys. Rev. E 73, 046104 (2006).

[14] P. Reimann, Phys. Rev. Lett. 86, 4992 (2001).

[15] V. M. Rozenbaum, T. Ye. Korochkova, A. A. Chernova, and M. L. Dekhtyar, Phys. Rev. E 83, 051120 (2011).

[16] H. Hagman, M. Zelan, and C. M. Dion, J. Phys. A 44, 155002 (2011). 